given access to dialysis, are suspected to have lower PD utilization.

Objectives Establish baseline PD utilization rates amongst patients stratified by funding status, design an intervention to improve PD utilization, and assess impacts of interventions on utilization rates.

Methods The intervention was designed by using interview data to identify patient and provider barriers to selection of PD and stratifying the top failure causes through failure modes and effects analysis (figure 1). PD utilization rates before and after the intervention were determined through a retrospective chart review. The impact of the intervention on patient understanding was assessed with patient surveys.

Results The chosen intervention was to revise educational materials to address common misconceptions. Prior to the class update, the utilization rate for $\mathrm{PD}$ was $14.3 \%$ in the non-traditionally funded group, primarily containing undocumented immigrants, compared to a $21.0 \%$ utilization rate in the traditionally funded group. After the updated class was implemented, PD utilization in increased to $26.1 \%$ and $22.9 \%$ in the non-traditionally and traditionally funded groups, respectively (figure 2-4). Preliminary data from post-class patient surveys suggests that patients with a better understanding of class material are more likely to make an earlier selection of a dialysis modality.

Conclusions Addressing patient misconceptions about peritoneal dialysis by updating educational materials is an

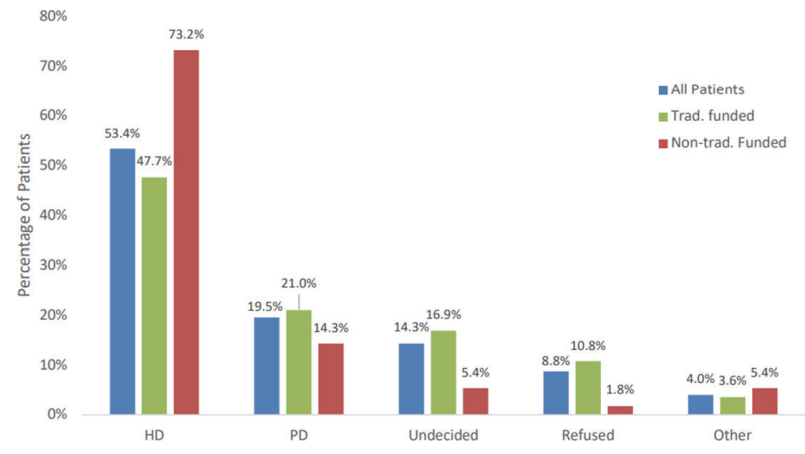

Abstract 19 Figure 2 Dialysis modality preferences for all patients at the Advanced Stage CKD clinic compared to patients who may qualify for outside funding and those who utilize traditional funding, prior to interventions, from 03/14/19-07/14/19. $n=251$ for all patients, $n=195$ for traditionally funded. $n=56$ for non-traditionally funded

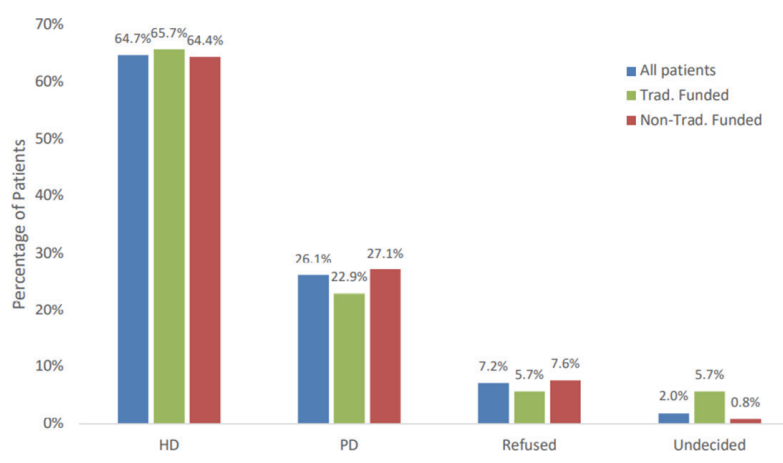

Abstract 19 Figure 3 Dialysis modality preference for patients at the ASCKD clinic from 07/2020-10/2020, after the intervention. $n=153$ for all patients, $n=118$ for traditional funding, $n=35$ for non-traditional funding

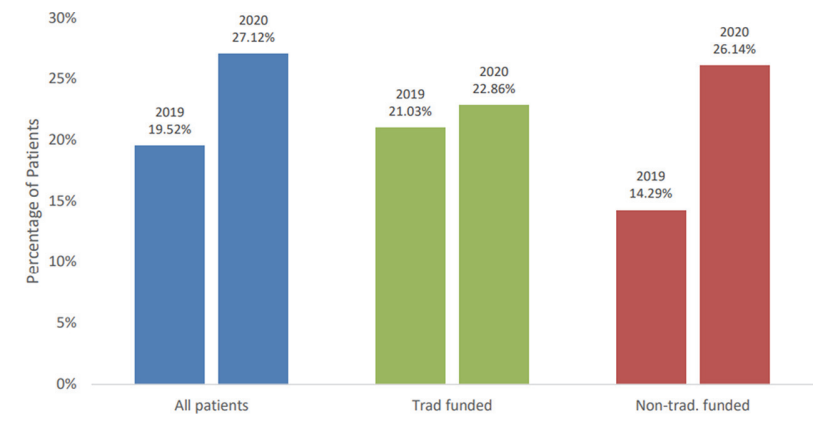

Abstract 19 Figure 4 Peritoneal dialysis (PD) utilization rates at the ASCKD clinic at Parkland hospital from 2019-2020, stratified by funding status

intervention that can increase the utilization rates of PD in patients with non-traditional funding. External factors could have played a role in increased PD utilization and additional data is needed to establish a more robust correlation.

\section{DIFFERENTIAL PERFORMANCE OF PEDIATRIC KEY PERFORMANCE INDICATORS BY HEALTH EQUITY FACTORS}

Lane Donnelly, Matthew Wood, Jean Chantra, Ling Loh, Brendan Burkart, Kristie Tan. Stanford University and Stanford Children's Health

\subsection{6/bmjoq-2021-IHI.20}

Background Growing literature shows that a number of social factors can be predictors of health outcomes - with influence on health issues such as infant mortality, surgical outcome, and wait times for kidney transplantation.

Objectives We evaluated the influence of multiple social factors on performance on multiple key performance indicators (KPIs) tracked at our pediatric healthcare system.

Methods We compared performance for rates on the following KPIs for a 2-year period $(2019,2020)$ - Serious Safety Events (SSE), Central Line Associated Blood Stream Infection (CLABSI), Hospital-Acquired Pressure Injury (HAPI), Codes Outside ICU, and Influenza Non-vaccination. We evaluated differential performance on those rates by the following factors that might affect health equity - patient gender, language preference, health insurance payer category, race and ethnicity, and estimated median household income based on zip code analysis - by creating simultaneous 95\% confidence intervals using the Wilson method with continuity correction and a Bonferroni adjustment for the number of categories compared. Results Children who resided in an area with a lower median household income had a statistically significant greater chance to develop HAPI (figure 1). A similar but not statistically significant trend was also seen with CLABSI. The SSE rate was 2.3 times higher in Spanish-speaking as compared to Englishspeaking children and 2.2 times higher for Medicaid as compared to commercially ensured patients (not statistically significant). Statistically significant differences in influenza nonvaccination rate were present for the following indicators: Spanish and Chinese Dialects $>$ than English speaking; Hispanic $>$ many other race and ethnicities; Commercial $<$ Public or Self-pay; and lower < higher median household income. Conclusions Factors influencing health equity correlated with decreased performance on a number of our health system KPIs. This quality and safety issue will benefit from 
FY19-FY20 Hospital Acquired Pressure Injury (HAPI) Rate Patient Demographics (Pediatric only)

Median Household Income among Patients with California Zip Codes (95,883 Patient Days 57\% of total)

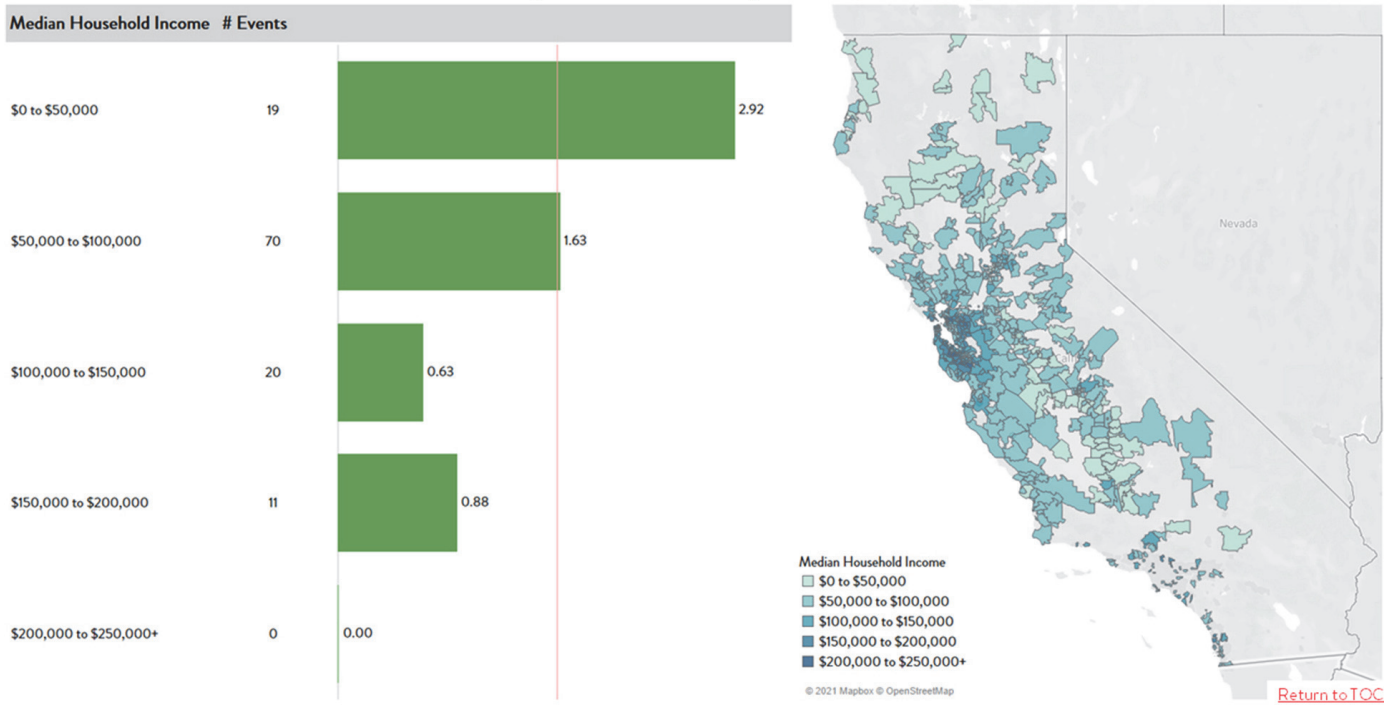

Abstract 20 Figure 1 Left shows the rate of hospital acquired pressure injuries separated by median household income. Right shows median household income by county for counties from which a patient visited our health system

improvement initiatives that seek to better understand and decrease the influence of these factors.

\section{IMPLEMENTING VIRTUAL CARE PLANNING WITH RESIDENT/FAMILY: A CANADIAN COVID LONG-TERM CARE EXPERIENCE}

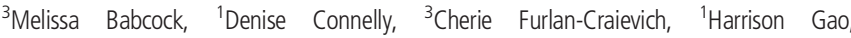
${ }^{1}$ Anna Garnett, ${ }^{7}$ Pam Hamilton, ${ }^{1}$ Melissa Hay, ${ }^{2}$ Lillian Hung, ${ }^{4}$ Jacqueline Ripley, ${ }^{6}$ Samantha Salatino, ${ }^{5}$ Lori Schindel Martin, ${ }^{4}$ Shannon Snelgrove, ${ }^{6}$ Nancy Snobelen. ${ }^{1}$ Western University, London, Canada; ${ }^{2}$ University of British Columbia, Vancouver, Canada; ${ }^{3}$ Vision 74, Sarnia, Canada; ${ }^{4}$ Copper Terrace, Chatham-Kent, Canada; ${ }^{5}$ Ryerson University, Toronto, Canada; ${ }^{6}$ WeRPN, Toronto, Canada; ${ }^{7}$ PIECES Canada, Toronto, Canada

\subsection{6/bmjoq-2021-|HI.21}

Background COVID-19 imposed extreme constraints on Canadian long-term care (LTC) homes, leading to intense isolation for residents, restricted family visits, and staff shortages. Consequently, these challenges negatively impacted the mental and physical health of residents, family, and interdisciplinary workforce in LTC homes.

Objectives

1. To describe how two LTC homes addressed promising practices - Presence of family, People in the workforce, and Future COVID-19 and non-COVID-19 care with implementation science.

2. To understand the enablers, barriers and outcomes to the implementation of an innovative re-engineered intervention P.I.E.C.E.S. ${ }^{\mathrm{TM}}$ (PIECES), designed to include families virtually for team-based resident care planning, and empower Registered Practical Nurses (RPNs) to build resilience and wellbeing.

Methods An interdisciplinary team (residents and family, nurses, and academic researchers), guided by the Consolidated Framework for Implementation Research, employed a mixedmethod design to investigate implementation processes, determinants, and outcomes. Interviews with residents/families and staff focus groups provided insight into enablers and challenges. Pre- and post-intervention results of the Connor Davidson Resilience Scale, Resilience at Work, and Assessment of Interprofessional Team Collaboration Scale informed understandings of personal, professional and organizational resilience outcomes.

Results Findings highlighted how implementation of virtual PIECES helped sustain enhanced person-centered care through more comprehensive, collaborative, efficient and effective teams, leading to better outcomes for residents. Moreover, results suggested improved resilience, wellbeing, and communication between LTC home staff and family. New learnings improved preparedness for future outbreaks. Providing a plan for adapting, embedding, and sustaining the intervention based on implementation science will accelerate the spread of highquality actionable research evidence.

Conclusions This is the first study to explore implementation processes of a virtually delivered PIECES intervention with meaningful engagement of multiple stakeholders (residents, family, RPNs). Findings provide evidence supporting important healthcare improvements, future spread of virtual interventions, and practice and policy changes for the LTC home sector.

\section{WELL BEGUN IS HALF DONE}

${ }^{1}$ Jason Boulanger, ${ }^{2}$ Evan Benjamin, ${ }^{2}$ Rachel Moyal-Smith, ${ }^{2}$ Patricia Folcarelli. ${ }^{1}$ CRICO; ${ }^{2}$ Ariadne Labs

\subsection{6/bmjoq-2021-IHI.22}

Background While many patient safety initiatives have successfully reduced harm in individual facilities and organizations, implementation, sustainment, and spread of these initiatives lags across the healthcare system.

Objectives To address this implementation gap, we created the Patient Safety Adoption framework, identifying and providing guidance for achieving the key elements of implementation (figure 1).

Methods The Framework for Implementation describes the required strategy and structure to achieve effective 